\title{
Investigation of the Crystallization Process in 2 nm CdSe Quantum Dots
}

\author{
Xiaobo Chen, Yongbing Lou, Anna C. S. Samia, Clemens Burda* \\ Center for Chemical Dynamics and Nanomaterials Research \\ Department of Chemistry, Case Western Reserve University, \\ 10900 Euclid Avenue, Cleveland, Ohio, 44106-7078 \\ E-mail: burda@case.edu
}

\section{Supporting Information}

XRD patterns of the CdSe NPs formed for 10 min and 90 min (Figure S1 ). 


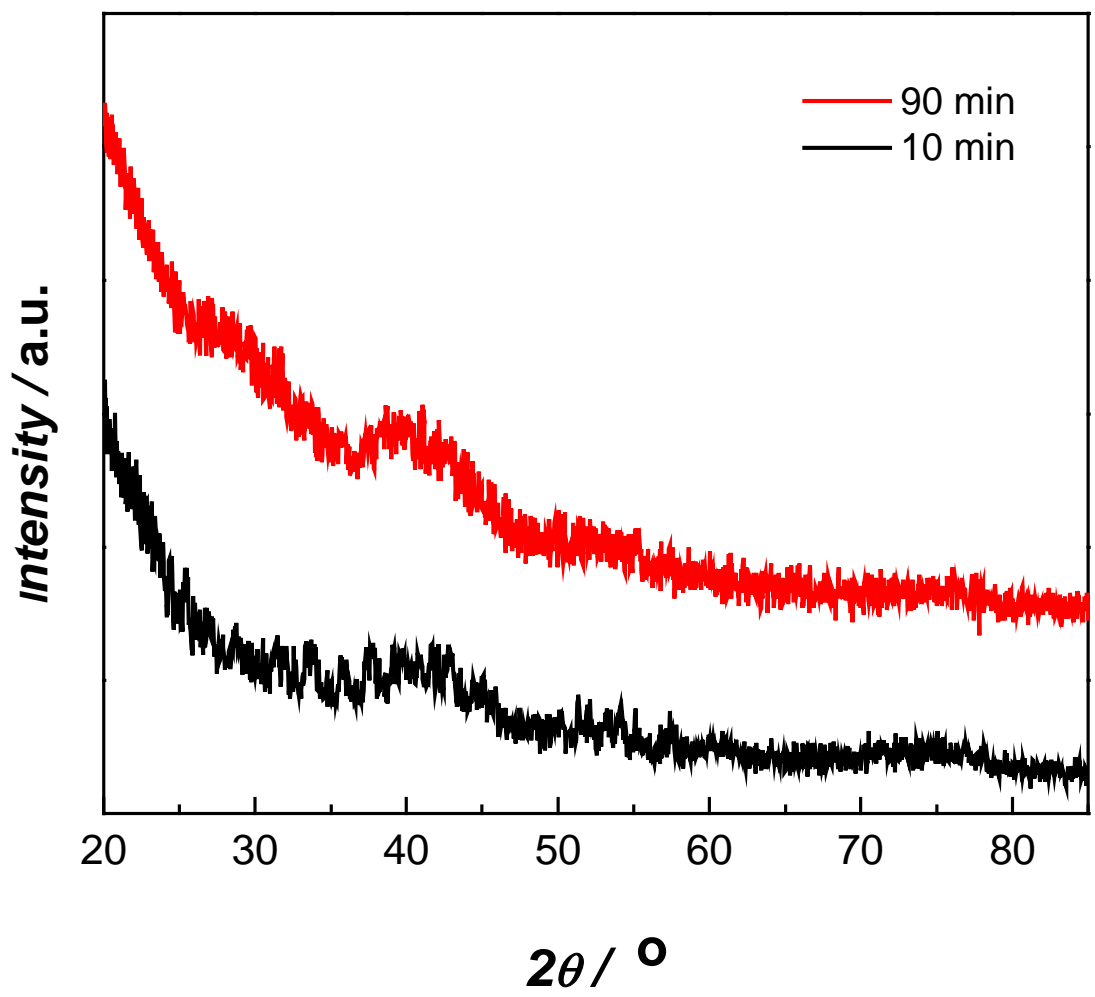

Figure S1. XRD patterns of the CdSe NPs formed for $10 \mathrm{~min}$ and $90 \mathrm{~min}$. Due to the small size of these samples, the XRD signal were weak for both samples. The XRD results shows that these CdSe NPs had similar sizes around $2.1 \pm 0.1 \mathrm{~nm},{ }^{\text {Ref }}$ and the CdSe NPs formed after 90 min's reaction had better crystallinity from their stronger diffraction pattern.

Ref: Murray, C. B.; Kagan, C. R.; Bawendi, M. G. Annu. Rev. Mater. Sci. 2000, 30, 545. 